\title{
Prognosis Value of microRNA-3677-3p in Lung Adenocarcinoma and Its Regulatory Effect on Tumor Progression
}

\author{
Jian Zhao',* \\ Hanbing Yu ${ }^{1}, *$ \\ Tianci Han ${ }^{1}, *$ \\ Xiangyu Zhu ${ }^{2}$
}

'Third Department of Thoracic Surgery, Liaoning Cancer Hospital and Institute, Shenyang, Liaoning, People's Republic of China; ${ }^{2}$ Department of General Medicine, Liaoning Cancer Hospital and Institute, Shenyang, Liaoning, People's Republic of China

*These authors contributed equally to this work
Correspondence: Xiangyu Zhu Department of General Medicine, Liaoning Cancer Hospital and Institute, Shenyang, Liaoning, People's Republic of China

$\mathrm{Tel} / \mathrm{Fax}+86-24-31916684$

Email zhuxiangyu_In@I63.com
Purpose: Lung adenocarcinomas (LUAD) was the most common subtype of lung cancer, and may result in a poor prognosis. This study was designed to explore the role of miR-3677$3 p$ in LUAD and discuss in what way it functions in LUAD.

Materials and Methods: We used RT-qPCR method to detect the expression levels of miR-3677-3p in 105 pairs of LUAD tissues and noncancerous tissues, as also as in LUAD cells. We used $\chi^{2}$ test to analyze the correlation between miR-3677-3p level and the clinical data. The prognosis significance of miR-3677-3p was inferred with Kaplan-Meier and multivariate Cox regression assays. Biological functions of LUAD cells were accessed by cell counting kit-8, transwell migration and invasion assay. The target gene of miR-3677-3p was investigated by luciferase activity assay.

Results: miR-3677-3p represented an ascendant expression in LUAD tissue specimens and cells. miR-3677-3p expression was associated with the TNM stage and with solitary metastasis. Over-expression of miR-3677-3p can shorten the overall survival period of LUAD patients when compared with low expression. Knockdown of miR-3677-3p suppressed the biology function of NSCLC cells including proliferation, migration, and invasion. KLF12 was a target gene of miR-3677-3p.

Conclusion: miR-3677-3p represents as a potential prognostic biomarker for LUAD. miR$3677-3$ p can promote LUAD progression by targeting KLF12.

Keywords: miR-3677-3p, lung adenocarcinomas, prognosis, $K L F 12$

\section{Introduction}

Carcinoma of the lung is one of the major causes in cancer-related mortality. To make matters worse, incidence and mortality of lung cancer are rising. ${ }^{1}$ In China, lung cancer ranked the first commonly diagnosed cancer in males (21.9\% of total cases), and second female (13.3\% of total cases) in 2018, which is expected to rise because of aging and growing population. ${ }^{2}$ Histologically, lung cancer is classed into two main types as small cell lung carcinoma (SCLC) and non-small-cell lung carcinoma (NSCLC). In these types, NSCLC accounts for $85 \%$ of all lung cancer cases, while its main subtype, lung adenocarcinoma (LUAD), comprises around $40 \%{ }^{3}$ Despite there were improvements in outcomes of lung cancer due to recent treatment advances, the 5-year survival only improved by small account and remains largely influenced by the stage when first diagnosed. ${ }^{4}$ Therefore, more research should be focused on screening reliable prognosis factors for LUAD. 
Cancers are characterized as complex heterogeneous diseases and are usually associated with genetic and epigenetic aberrations. ${ }^{5}$ MicroRNAs (miRNAs), though small in length (about 19-24 nucleotides), can function as gene expression regulators in the posttranscriptional process by binding to target sequences in messenger RNA (mRNA) complementarily, thereby regulating the production of the protein. ${ }^{9}$ Advanced technologies lead to simultaneous detection of multiple genes over some time points, and revealed the alterations of miRNA expression are the rule rather than exceptional in human cancer. ${ }^{10}$ Because of the ability in cellular processes regulation, miRNAs were hypothesized to predict cancer prognosis, and then many of them have been verified successfully as prognostic markers that predict cancer outcome. ${ }^{10}$ Till now, a lot of reports supported the clinical significance of miRNAs as prognostic biomarkers in various cancers. For instance, in gastric cancer, a fivemiRNA signature can accurately predict the prognosis of gastric cancer. ${ }^{11}$ Similarly, a 7-miRNA signature has been identified to be associated with survival in nivolumabtreated lung cancer patients. ${ }^{12}$ microRNA-3677-3p (miR-3677-3p) has been investigated as an oncogene of breast cancer, ${ }^{13}$ a useful factor to predict tumor mutation burden level in endometrial cancer, ${ }^{14}$ and a prognostic miRNA for hepatocellular carcinoma. ${ }^{15}$ However, the prognostic power of miR-3677-3p in LUAD has not been validated.

Kruppel Like Factor 12 (KLF12), a family of DNA binding transcriptional regulators, has been reported to be downregulated in lung carcinoids, especially lower in samples with positive nodal status and with necrosis. ${ }^{6}$ KLF12 was involved in the regulation of many cellular processes, so its dysregulation led to different roles in different neoplasm, as tumor-suppressive or -oncogenic gene. ${ }^{7,8}$ More importantly, KLF12 was found to act as a tumor suppressor in lung carcinoids and low expression of KLF12 has been verified to be associated with shorter survival in lung cancer. $^{7}$ It has been reported that KLF12 overexpression can abrogate the proangiogenic effect of miR-141 on human umbilical vein vascular endothelial cells in small cell lung cancer. ${ }^{16}$

The focus of this study was on the prognostic value of miR-3677-3p in LUAD and regulation in cancer cellular processes. We first determine the expression of miR-3677$3 p$ in LUAD tissues and cells, then evaluated its potential prognostic significance based on the clinical data. In addition, we accessed the regulatory effect on tumor progression, and explored the preliminary mechanism by which miR-3677-3p regulated KLF12.

\section{Materials and Methods}

\section{Tissues and Cells}

This study was approved by the Ethics Committees of Liaoning Cancer Hospital and Institute, and conducted in accordance with the Declaration of Helsinki. All patients provided written informed consent before inclusion. Patients were selected from LUAD patients receiving surgical treatment at Liaoning Cancer Hospital and Institute from April 2012 to October 2015. The patients who received any type of therapy for cancer before surgery were excluded. Based on this, 105 paired tissues with adequate clinical and histological characteristics were collected in the study according to the criteria proposed by the IASLC/ATS/ERS in 2011. ${ }^{17} \mathrm{TNM}$ stage was determined according to the 7 th edition of the American Joint Committee on Cancer (AJCC) cancer staging manual.

Four kinds of human LUAD cells, A549, HCC827, NCI-H441, and NCI-H1734, and an immortalized human bronchial epithelial cell line (BEAS-2B) were obtained from ATCC (Rockville, MD, USA) and cultured in RPMI medium 1640 (Invitrogen, USA) supplemented with $10 \%$ FBS (Invitrogen, USA). All cells were maintained at the condition of $5 \% \mathrm{CO}_{2}$ and $37^{\circ} \mathrm{C}$.

\section{Cell Transfection}

miR-3677-3p inhibitor (miR inhibitor), miR-3677-3p inhibitor control (inhibitor NC), the miR-3677 sequence (miR mimic) and mimic NC, siRNA negative control (si-NC), siRNA targeted to KLF12, pcDNA3.1 control, and pcDNA3.1-KLF12 was synthesized by Ribobio. Co., (Guangzhou, China). Firstly, cells were seeded at $50 \%$ confluence without antibiotics one day before transfection. The transfection was conducted using Lipofectamine 3000 (Invitrogen) according to the manufacturer's direction. After transfection, the cells were incubated for $24 \mathrm{~h}$ or $48 \mathrm{~h}$ for the next step.

\section{Dual-Luciferase Reporter Assay}

3'-UTR regions from KLF12 containing the predicted seeding sequences were used to construct the dualluciferase reporter vectors of WT-KLF12, and the mutant one that lacks GGGUGCU was inserted into the pGL3.0 vector which expressed firefly luciferase as MUT-KLF12. 
A549 cells were transfected with WT-KLF12 or MUTKLF12, together with miR inhibitor or inhibitor NCby Lipofectamine 3000 transfection reagent. $48 \mathrm{~h}$ later, cell lysates were harvested and then reseeded into 96-well plates. The plasmid expressing Renilla luciferase was used as the internal reference. Luciferase activity was measured with a SpectraMax iD5 Multi-Mode Microplate Reader (Molecular Devices, USA).

\section{RNA Isolation and Real-Time Quantitative PCR (RT-qPCR) Assay}

Total RNA was extracted from LUAD tissue homogenates and cultured cells by the RNeasy Mini Kit (Qiagen, China). For target KLF12 mRNA detection, genomic DNA was removed RNA was isolated using the RNase-Free DNase Kit (Qiagen, China), and then reverse-transcribed using iScript cDNA Synthesis Kit (Bio-Rad, USA) based on the manufacturer's recommendations. For miR-3667-3p detection, mirVana miRNA isolation kit (Life Technologies, USA) was used following the manufacturer's instructions. Sample cDNA was amplified and quantified in a Thermal Cycler Dice $^{\text {TM }}$ Real-Time System III (Takara, Japan). Levels of $K L F 2$ were normalized to glyceraldehyde-3-phosphate dehydrogenase (GAPDH), and levels of miR-3677-3p were normalized to U6. Data were analyzed via $2^{-\Delta \Delta \mathrm{Ct}}$.

\section{Cell Proliferation Assay}

The proliferation rate was measured using a cell proliferation assay kit (CCK-8, Dojindo Corporation, Japan) following the manufacturer's handbook. Briefly, cells $\left(4 \times 10^{3}\right.$ cells $)$ cultured in a 96-well plate were added with $10 \mu \mathrm{L}$ of CCK- 8 solution every $24 \mathrm{~h}$ in $72 \mathrm{~h}$ and then incubated for $2 \mathrm{~h}$ in a $\mathrm{CO}_{2}$ incubator. The subsequent absorbance change was measured using a microplate reader (Molecular Devices, USA) at $450 \mathrm{~nm}$.

\section{Cell Migration and Invasion Assays}

The migration and invasiveness of LUAD cancer cells were assessed by the number of cells that migrated or invaded through uncoated transwell chambers (Corning, USA) or Matrigel (Corning, USA)-coated ones. LUAD cells $\left(1 \times 10^{5}\right.$ cells/well $)$ were seeded in the upper chambers without serum and incubated for $24 \mathrm{~h}$. The lower chambers were filled with medium with $10 \%$ FBS. Cancer cells that migrated or invaded to the lower surface were fixed with $70 \%$ ethanol, stained, and five random fields of the A549 and HCC827 cells' image were counted under a light microscope (Nikon, Japan).

\section{Western Blot Analysis}

Briefly, A549 cells were homogenized in ice-cold RIPA buffer mixing with PMSF (MP Biomedicals, Solon, OH, USA). After $30 \mathrm{~min}$ of incubation on ice and brief sonication, protein concentrations were determined using BCA protein assay kit (Thermo Scientific, USA). The assay was performed as described previously, ${ }^{18}$ with KLF12 primary antibody and GADPH primary antibody (as control). Immunoreactive proteins were measured using eZwest Lite Auto Western Blotting System (GenScript, Nanjing, China).

\section{Target Gene Identification}

To predict target genes regulated by miR-3677-3p, we used miRDB, miRWalk, and TargetScan methods. The predicted target genes of miR-3677-3p supported by all the three methods were subjected to literature retrieval and the first downregulated gene in LUAD was selected for further analysis.
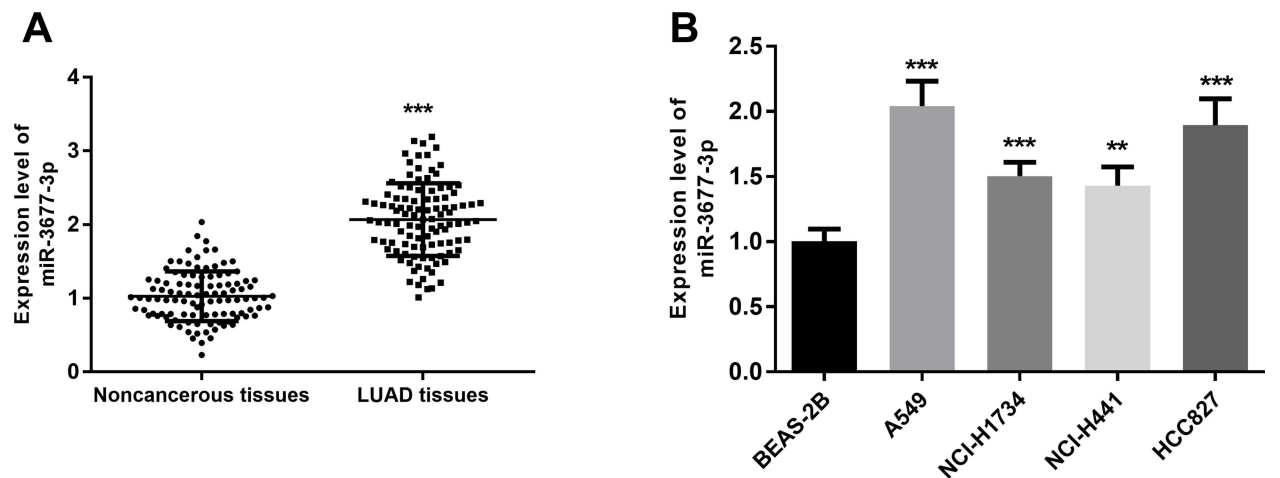

Figure I Expression level of miR-3677-3p in LUAD tissue samples and cells. (A) miR-3677-3p is upregulated in LUAD tissue samples and non-cancerous samples; (B) expression level of miR-3677-3p in NSCLC cell lines (A549, HCC827, NCl-H44I, and NCl-HI734). **P $<0.0 \mathrm{I}, * * * P<0.00 \mathrm{I}$. 
Table I Correlation of the miR-3677-3p Expression with Clinical Characteristics in LUAD

\begin{tabular}{|c|c|c|c|c|}
\hline \multirow[t]{2}{*}{ Parameters } & \multirow[t]{2}{*}{ Cases $(n=105)$} & \multicolumn{2}{|c|}{ miR-3677-3p Expression } & \multirow[t]{2}{*}{$P$} \\
\hline & & Low $(n=48)$ & High $(n=57)$ & \\
\hline \multicolumn{5}{|l|}{ Age } \\
\hline$\leq 55$ & 51 & 22 & 29 & 0.606 \\
\hline$>55$ & 54 & 26 & 28 & \\
\hline \multicolumn{5}{|l|}{ Gender } \\
\hline Female & 57 & 24 & 33 & 0.419 \\
\hline Male & 48 & 24 & 24 & \\
\hline \multicolumn{5}{|l|}{ Tumor size } \\
\hline$\leq 3 \mathrm{~cm}$ & 56 & 30 & 26 & 0.084 \\
\hline$>3 \mathrm{~cm}$ & 49 & 18 & 31 & \\
\hline \multicolumn{5}{|l|}{ ISmoking status } \\
\hline Non-smoker & 52 & 27 & 25 & 0.206 \\
\hline Smoker & 53 & 21 & 32 & \\
\hline \multicolumn{5}{|l|}{ Differentiation } \\
\hline Well, Moderate & 53 & 25 & 28 & 0.762 \\
\hline Poor & 52 & 23 & 29 & \\
\hline \multicolumn{5}{|c|}{ Lymph node metastasis } \\
\hline Negative & 65 & 36 & 29 & 0.011 \\
\hline Positive & 40 & 12 & 28 & \\
\hline \multicolumn{5}{|l|}{ TNM stage } \\
\hline I, II & 68 & 36 & 32 & 0.044 \\
\hline III, IV & 37 & 12 & 25 & \\
\hline \multicolumn{5}{|l|}{ Solitary metastasis } \\
\hline Negative & 93 & 46 & 47 & 0.032 \\
\hline Positive & 12 & 2 & 10 & \\
\hline
\end{tabular}

\section{Statistical Analysis}

Data were expressed as means \pm standard deviation (SD) from at least 3 independent experiments. The statistical differences were analyzed by the one-way analysis of variance (ANOVA) or the two-tailed Student's $t$-tests. $\chi^{2}$ test was used to compare the association between miR3677-3p expression and clinical data of patients. KaplanMeier curve with Log rank test and multivariate Cox regression assay was performed to access the prognostic significance of miR-3677-3p. $P<0.05$ indicates a statistical significance.

\section{Results}

\section{Expression of miR-3677-3p and}

\section{Correlation with Clinicopathologic}

Features in LUAD

miR-3677-3p expression in 105 human LUAD tissues and their adjacent noncancerous tissues was investigated by RT-qPCR. In contrast to the weak expression of miR- 3677-3p in normal tissues and cells, a high level of miR3677-3p was detected in LUAD tissues and cells $(P<0.01$ or $P<0.001$, Figure 1). The enrolled 105 patients with LUAD were grouped into low miR-3677-3p expression $(\mathrm{n}=48)$ and high miR-3677-3p expression $(\mathrm{n}=57)$ according to the mean of miR-3677-3p expression value in the enrolled resected tumor tissues. Table 1 showed the association between events of poor prognosis and miR3677-3p expression. miR-3677-3p expression was associated with TNM stage $(P=0.044)$, and with solitary metastasis $(P=0.032)$.

\section{Multivariate Analysis and Kaplan-Meier Plotter for Prognoses of Patients with LUAD}

Multivariate Cox regression was used to estimate hazard ratios (HRs) and 95\% confidence intervals (CIs) of the association between miR-3677-3p expression and overall survival. As shown in Table 2, the analysis revealed that 
Table 2 Multivariate Cox Analysis of Clinical Characteristics Correlated to Overall Survival

\begin{tabular}{|l|c|c|c|}
\hline \multirow{2}{*}{ Characteristics } & \multicolumn{3}{|c|}{ Multivariate Analysis } \\
\cline { 2 - 4 } & HRs & $95 \%$ Cls & $P$ \\
\hline miR-3677-3p & 2.575 & $1.166-4.368$ & 0.016 \\
Age & 1.325 & $0.724-2.424$ & 0.361 \\
Gender & 1.395 & $0.768-2.534$ & 0.274 \\
Tumor size & 1.099 & $0.602-2.005$ & 0.758 \\
Smoking status & 1.324 & $0.71 \mathrm{I}-2.467$ & 0.376 \\
Differentiation & 1.082 & $0.573-2.042$ & 0.808 \\
Lymph node metastasis & 1.867 & $0.96 \mathrm{I}-3.627$ & 0.066 \\
TNM stage & 2.327 & $1.093-4.954$ & 0.028 \\
Distant metastasis & 3.450 & $1.150-10.348$ & 0.027 \\
\hline
\end{tabular}

Abbreviations: HRs, hazard ratios; Cis, confidence intervals; TNM, tumor, node, metastasis.

up-regulation of miR-3677-3p was a significant independent prognostic factor for LUAD patients (HR, 2.575; 95\% CI, 1.166-4.368, $P=0.016$ ). To predict the prognosis of patients with LUAD, a Kaplan-Meier Plotter was built to predict overall survival difference among patients with different miR-3677-3p level. The patients with high miR3677-3p expression tended to exhibit a shorter overall survival compared with that in patients with low expression (Figure 2, Log rank test $P=0.026$ ).

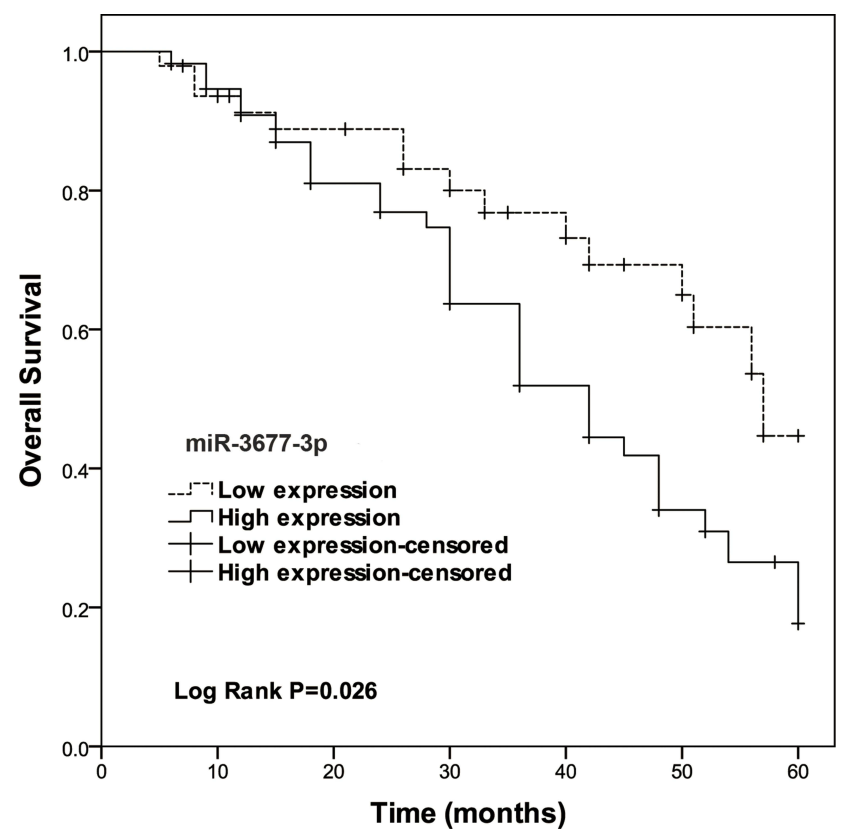

Figure 2 Comparison of 5-year survival curves of LUAD patients with high and low expression of miR-3677-3p by Kaplan-Meier plotter. (Log rank test $P=0.026$ ).

\section{miR-3677-3p Expression Influence LUAD} Cell Proliferation, Migration, Invasion

The role of miR-3677-3p in LUAD cell proliferation, migration, and invasion was analyzed by transfecting A549 and HCC827 cells with miR-3677-3p inhibitor and mimics. miR-3677-3p inhibitor and mimics successfully resulted in a notable reduction or addition in the miR-3677-3p levels $(P<0.001$; Figure $3 \mathrm{~A}$ and $\mathrm{C})$. The results of the CCK- 8 assay indicated that A549 and HCC 827 cell proliferation was significantly inhibited by the miR-3677-3p inhibitor and promoted by the miR-3677-3p mimics $(P<0.01, P<0.001$; Figure 3B and $\mathrm{D})$. The Transwell assay evaluating the migration capability revealed that miR-3677-3p inhibitor suppressed cell migration, while mimic promotes migration $(P<0.001$; Figure 4A and B). Similarly, the data from the transwell assay demonstrated that the invasion ability in A549 and HCC827 cells was significantly suppressed by miR-3677-3p inhibitor and increased by miR-3677-3p mimic $(P<0.01, P<0.001$; Figure 4C and D). These mentioned findings suggested that miR$3677-3 p$ promotes the proliferation, migration, and invasion of LUAD.

\section{miR-3677-3p Targeted the 3'-UTR of KLFI2}

Bio-informatics prediction suggests that KLF12 (Gene ID: 11278) ranked first in the overlapped gene list from miRDB, miRWalk, and TargetScan. Combined with the downregulation of KLF12 in LUAD, miR-3677-3p may target the 3'-UTR of KLF12 in LUAD. A possible direct link between miR-3677-3p and KLF12 3'-UTR was examined using TargetScan (Figure 5A). The expression of $K L F 12$ in LUAD tissues was downregulated $(P<0.01$ or $P<0.001$; Figure 5B), negatively correlated with miR3677-3p expression $(P<0.001$; Figure 5C). Besides, KLF12 was downregulated in LUAD cells $(P<0.01$; Figure 5D), and silence or overexpression of miR-3677$3 p$ can lead to an increase or reduction of KLF12 mRNA level $(P<0.001$; Figure 5E). The luciferase activity results indicate that was transfection of the miR-3677-3p was lower after mimic transfected than in the control groups $(P<0.001$; Figure $5 \mathrm{~F})$. By using Western blot analysis, significant decrease in KLF12 was found in miR-3677-3poverexpressed A549 cells, while increase in KLF12 was found in miR-3677-3p-knockdown A549 cells, compared to untreated cells (Figure 5G). 
A
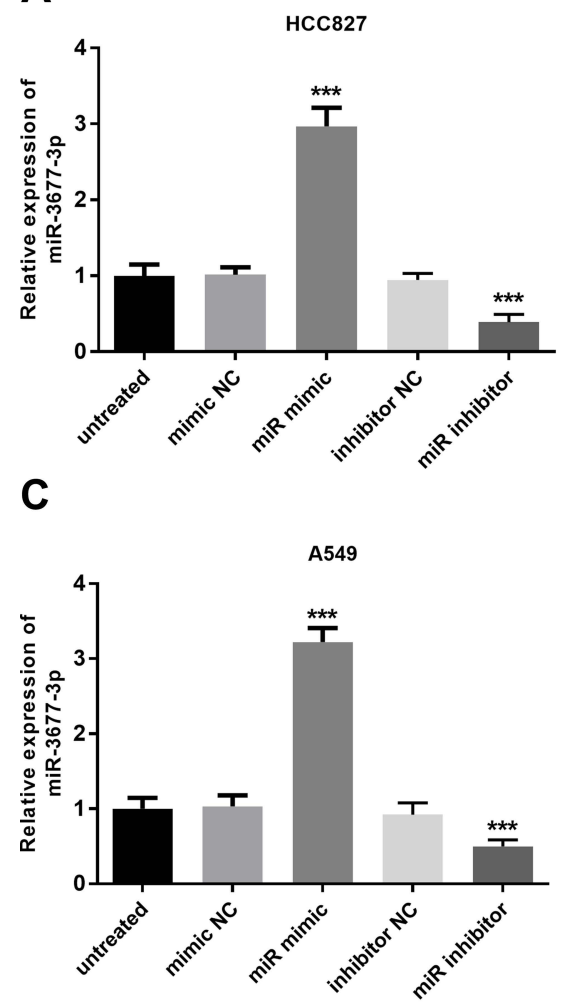

B

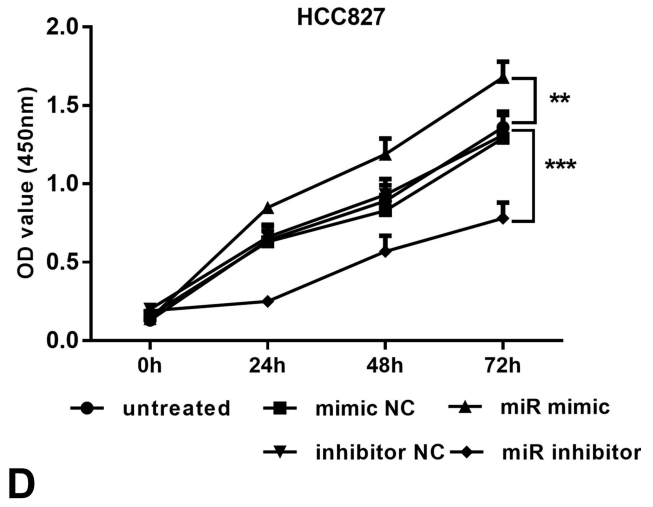

A549

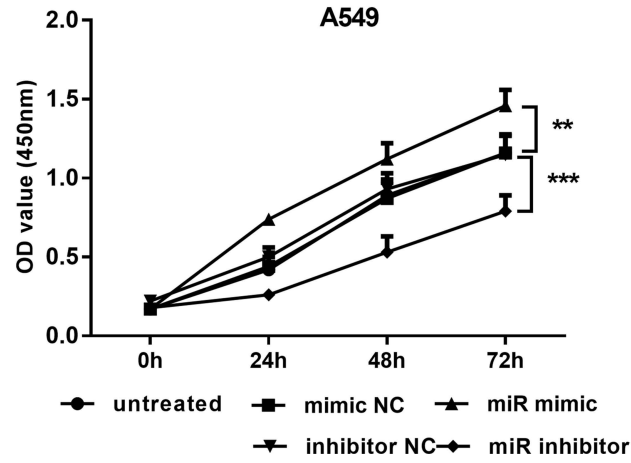

Figure 3 Proliferation of A549 and HCC827 cells were suppressed after miR-3677-3p silence and boosted by miR-3677-3p overexpression when compared with untreated cells. (A and $\mathbf{C})$ Expression level of miR-3677-3p was determined by RT-qPCR; (B and $\mathbf{D})$ proliferative capacity was measured by CCK-8. $* * P<0.01$, $* * * P<0.001$.
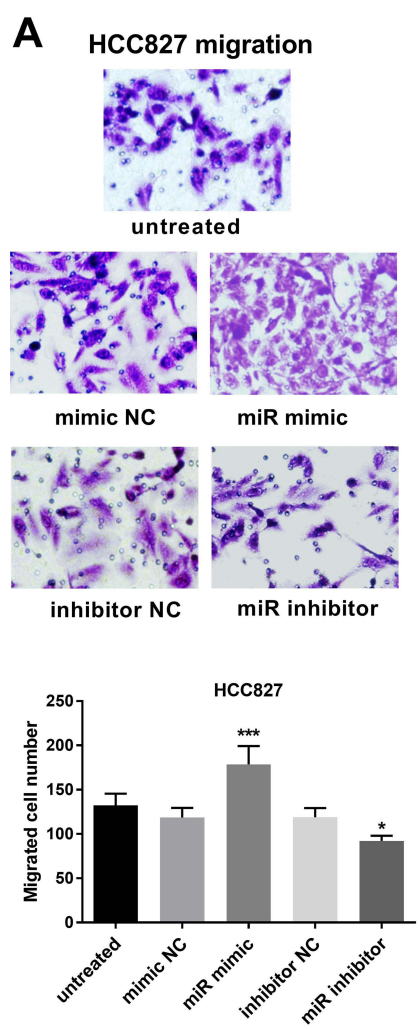

B
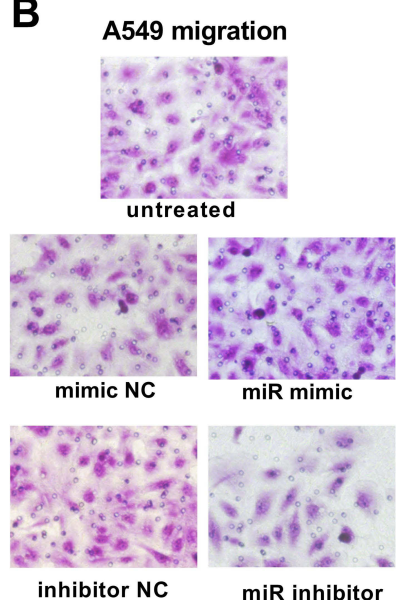
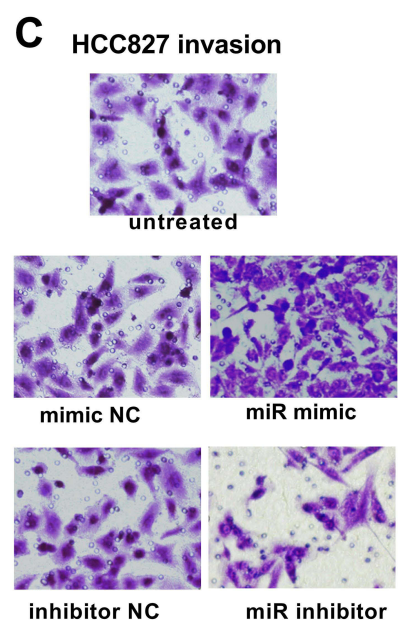

D A549 invasion

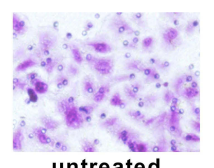

untreated
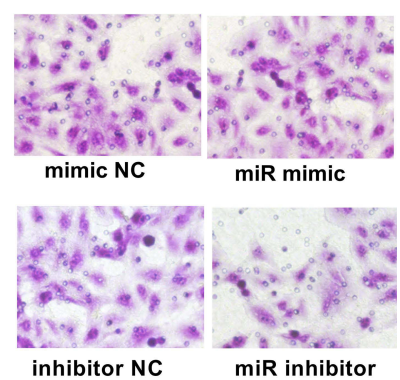
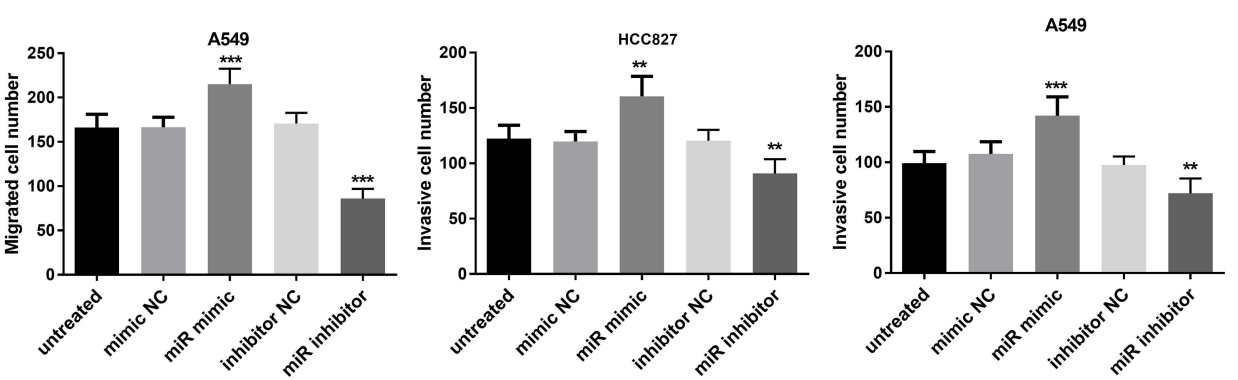

Figure 4 Migration, and invasion of A549 and HCC827 cells were suppressed after miR-3677-3p inhibition and enhanced by miR-3677-3p overexpression when compared to untreated cells. (A and $\mathbf{B})$ Migratory ability was measured by transwell assay; $(\mathbf{C}$ and $\mathbf{D})$ invasive ability was measured by modified transwell assay. $* P<0.0 \mathrm{I}$, $* * P<0.0 \mathrm{I}$, $* * * p<0.001$. 
A
Position 107-113 of KLF12 3' UTR 5' ...AAAGGGCAUCACCAUCCCACGAU ...
has-miR-3677-3p
MUT-KLF12
I | | |1।|1।|

\section{3' CCGGCACCGGUCUCGGGUGCUC}
5' aAaggCCAUGaCCUU GgGUGCUU

B
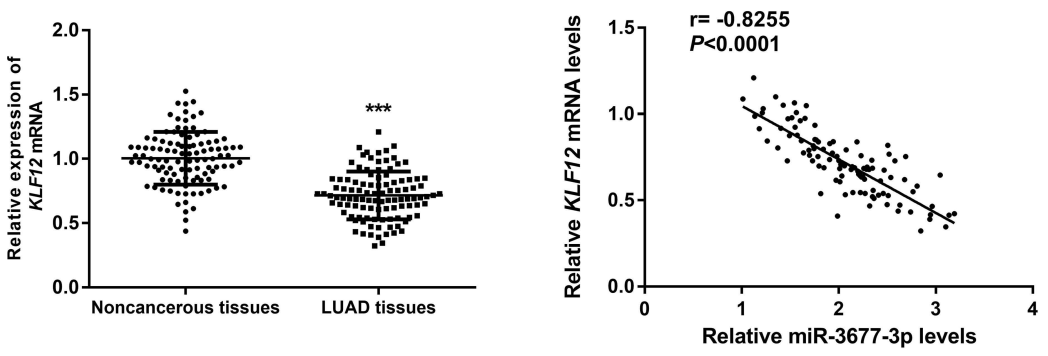

E

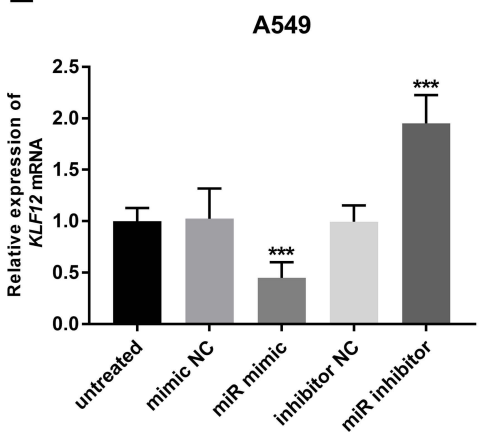

Figure 5 KLFI2 is a target of miR-3677-3p. (A) Predicted miR-3677-3p target sequence in the 3'-UTR of KLFI2; (B) KLFI2 mRNA were decreased in LUAD tissues; (C) KLFI 2 expression was negatively correlated with expression of miR-3677-3p. (D) KLFI2 mRNA were decreased in LUAD cells; (E) KLFI2 mRNA level was influenced by miR-3677-3p silence or overexpression. (F) Dual-luciferase reporter assay was performed in A549 cells. (G) Western blot analysis of protein levels of KLFI 2 and GADPH. $* * p<0.01$, $* * * P<0.001$.

These findings were further supported by rescue experiments using miR-3677-3p and siRNA of KLF12 $(P<0.001$; Figure 6A). Notably, inhibition of miR-3677$3 \mathrm{p}$ and $K L F 12$ can restore the decreased cell proliferation because of which miR-3677-3p inhibition $(P<0.01$; Figure 6B). Similarly, the suppression of cell migration and invasion caused by miR-3677-3p silence ascended after $K L F 12$ inhibition $(P<0.01$; Figure $6 \mathrm{C}$ and D).

\section{Discussion}

Recent studies have reported that miR-3677 is abnormally expressed in several malignant tumors and has a relation with cancer survival rate. ${ }^{19}$ For instance, miR-3677-3p has a higher expression in hepatocellular carcinoma tissues than adjacent non-tumor tissues and is negatively correlated with the overall survival of patients with hepatocellular carcinoma. ${ }^{20-22}$ In colon cancer patients, miR-3677 also represents an elevated expression and is verified to be an independent prognostic miRNA for patients' survival based on the 5-year survival rate. ${ }^{23}$ Our study provides evidence for miR-3677-3p upregulation in LUAD tissues and cells. Furtherly, upregulation of miR3677-3p expression was associated with the TNM stage and with distant metastasis in LUAD patients. Multivariate Cox regression revealed miR-3677-3p can act as a significant independent prognostic factor for LUAD patients. Kaplan-Meier Plotter verified the association between miR-3677-3p expression and overall survival, presenting as LUAD patients with higher miR-3677-3p expression result in a short survival. Another study about the identification of microRNAs as prognostic markers in LUAD patients also suggested that miR-3677 can develop 
A

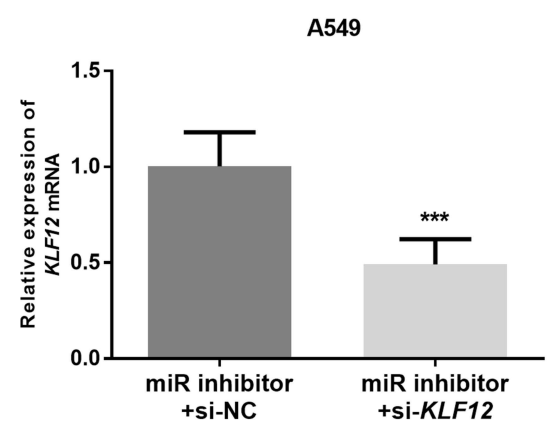

C
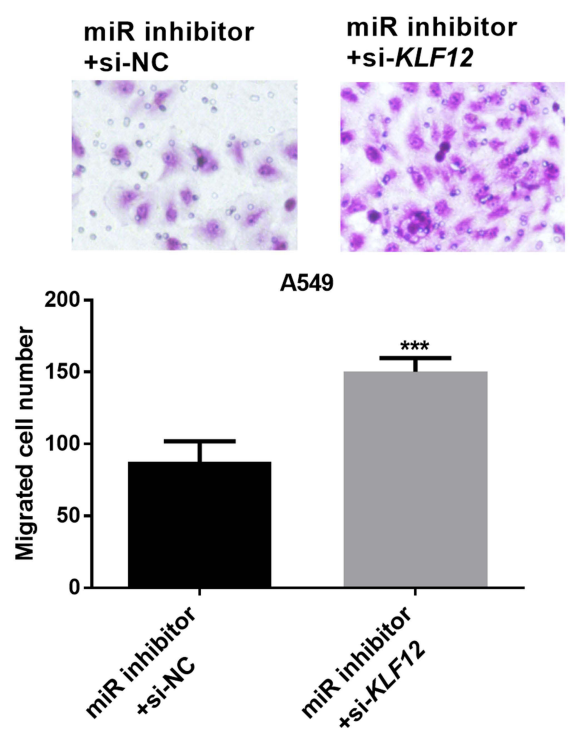

B

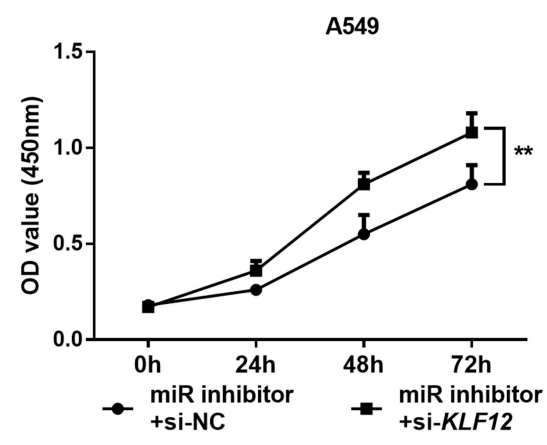

D

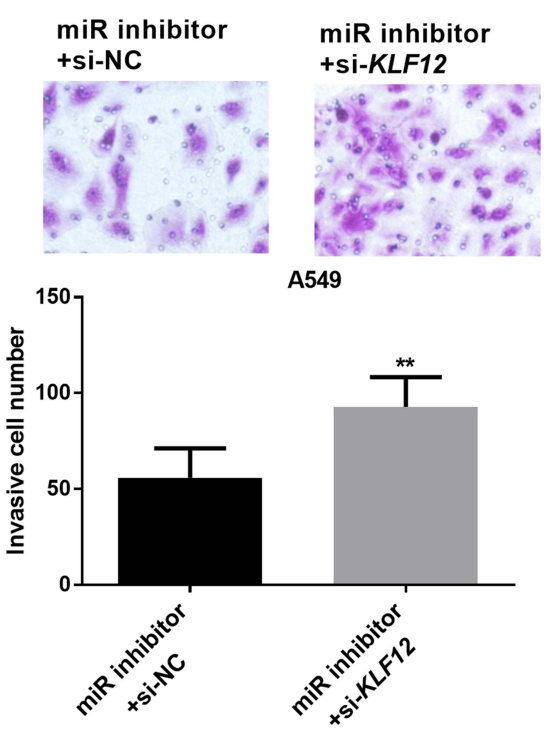

Figure 6 KLF/2 inhibition can rescue the suppression of cell proliferation, migration, invasion caused by miR-3677-3p. (A) RT-qPCR was performed to evaluate the expression level of KLFI 2 mRNA. (B) Interfering KLFI 2 reversed the effect of miR-3677-3p inhibitor on cell proliferation; (C and D) KLFI 2 inhibition reversed the effect of miR-3677-3p inhibitor on cell migration and invasion. $* * P<0.01$, $* * * P<0.001$.

to predict prognostic markers for LUAD according to their prognostic potential CWx scores. ${ }^{24}$ Combined with these results, miR-3677-3p has the potential to be a predictive factor for the prognosis of LUAD patients.

Cancers involve a set of essential cellular traits for tumor maintenance and malignant transformation, including sustained proliferative signaling and activation of invasion and metastasis. ${ }^{25}$ It is reported that miR-3677 acts oncogenic activity in breast cancer, and promoting breast cancer cell proliferation, migration, and invasion by inhibiting TLE3 expression. ${ }^{26}$ Since we found miR3677-3p was involved in a high-expression-related poor prognosis in LUAD patients, this indicates its oncogenesis function in LUAD. Thus, we further performed a series of experiment to study the cellular function and observed that miR-3677-3p upregulation can promote cell proliferation, migration, and invasion of LUAD cells. By contrast, inhibiting miR-3677-3p expression via miR-3677-3p inhibitor can weaken the proliferation, migration, and invasion of LUAD cells. This influence of miR-3677-3p on cell function was also found in hepatocellular carcinoma, presenting as influence in the proliferation and cell cycle. ${ }^{27}$ Moreover hypoxia-induced miR3677-3p up-regulation contributed to hepatocellular carcinoma malignancy and invasiveness by mediating SIRT5 inhibition, which could increase hepatocellular carcinoma cell proliferation, migration, and invasion in hypoxic microenvironments. ${ }^{28}$ In this study, we found miR3677-3p can function as a tumor promoter to benefit the proliferative capacity, migratory and invasive ability of LUAD cells, which provide clues to develop a new therapeutic target. 
As mentioned above, miR-3677-3p may play considerable roles in regulating LUAD progression, formation, or metastasis. ${ }^{29}$ But it is not known enough in the underlying molecular mechanisms. The most common mechanism of miRNAs function is binding to its target mRNA, leading to mRNA destabilization, degradation, and the resulting decrease in mRNA expression levels. ${ }^{30}$ For example, through targeting the $3^{\prime}$-UTR region of GSK $3 \beta$ or SIRT5, miR-3677-3p could inhibit GSK3 $\beta$ or SIRT5 expression and play a crucial role in hepatocellular carcinoma. ${ }^{27,28}$ Another study using the bioinformatics analysis found miR-3677-3p putatively targets 3'-UTR of SphK1 (at the position of 235242 ) and promotes OS cell progression in vitro. ${ }^{31}$ We performed the bioinformatics analysis, and found there are ten target sites between KLF12 and miR-3677-3p at 3'-UTR. Hence we deducted that miR-3677-3p might promote LUAD progression by modulating KLF12. The inverse expression level of miR-3677-3p and KLF12 primarily implied the binding, and the luciferase activity change with or without miR-3677-3p verified the direct binding relationship between miR-3677-3p and KLF12. Further, the rescue experiment showed KLF12 can reverse the effect of miR$3677-3 p$ on cell function. It has been reported that KLF12 could act as a tumor suppressor in the human lung cancer. ${ }^{7}$ In addition, miR-137, miR-141, and miR-200a-3p could target $K L F 12$ to act as tumor promoters in different cancers. ${ }^{32-34}$ Based on these studies, miR-3677-3p may target KLF12 and play roles in LUAD partly at least. Our study is currently the only one to verify the interaction between miR-3677-3p and KLF12, especially in LUAD.

\section{Conclusion}

In summary, miR-3677-3p was up-regulated in LUAD tissues and cells and was correlated with clinicopathological parameters, including TNM stage and distant metastasis. miR-3677-3p knockdown could alter the capabilities of proliferation, cell migration, and invasion in LUAD cell lines. miR-3677-3p might act as a tumor promoter in LUAD by targeting KLF12.

\section{Disclosure}

The authors report no conflicts of interest in this work.

\section{References}

1. Bade BC, Dela CCS. Lung cancer 2020: epidemiology, etiology, and prevention. Clin Chest Med. 2020;41(1):1-24. doi:10.1016/j. ccm.2019.10.001
2. Feng RM, Zong YN, Cao SM, Xu RH. Current cancer situation in China: good or bad news from the 2018 global cancer statistics? Cancer Commun. 2019;39(1):22. doi:10.1186/s40880-019-0368-6

3. Nasim F, Sabath BF, Eapen GA. Lung cancer. Med Clin North Am. 2019;103(3):463-473. doi:10.1016/j.mcna.2018.12.006

4. Bagcchi S. Lung cancer survival only increases by a small amount despite recent treatment advances. Lancet Respir Med. 2017;5 (3):169. doi:10.1016/S2213-2600(17)30041-3

5. Pagliarini V, Naro C, Sette C. Splicing regulation: a molecular device to enhance cancer cell adaptation. Biomed Res Int. 2015;2015:543067. doi:10.1155/2015/543067

6. Rapa I, Votta A, Giorcelli J, et al. Proposal of a panel of genes identified by miRNA profiling as candidate prognostic biomarkers in lung carcinoids. Neuroendocrinology. 2021;111(1-2):115-122. doi:10.1159/000506401

7. Godin-Heymann N, Brabetz S, Murillo MM, et al. Tumoursuppression function of KLF12 through regulation of anoikis. Oncogene. 2016;35(25):3324-3334. doi:10.1038/onc.2015.394

8. Ding L, Ding Y, Kong X, et al. Dysregulation of Krüppel-like factor 12 in the development of endometrial cancer. Gynecol Oncol. 2019;152(1):177-184.

9. Bhaskaran M, Mohan M. MicroRNAs: history, biogenesis, and their evolving role in animal development and disease. Vet Pathol. 2014;51 (4):759-774. doi:10.1177/0300985813502820

10. Iorio MV, Croce CM. MicroRNA dysregulation in cancer: diagnostics, monitoring and therapeutics. A comprehensive review. EMBO Mol Med. 2012;4(3):143-159. doi:10.1002/emmm.201100209

11. Zhang Z, Dong Y, Hua J, et al. A five-miRNA signature predicts survival in gastric cancer using bioinformatics analysis. Gene. 2019;699:125-134. doi:10.1016/j.gene.2019.02.058

12. Halvorsen AR, Sandhu V, Sprauten M, et al. Circulating microRNAs associated with prolonged overall survival in lung cancer patients treated with nivolumab. Acta Oncol. 2018;57(9):1225-1231. doi:10.1080/0284186X.2018.1465585

13. Martinez-Gutierrez AD, Cantú de León D, Millan-Catalan O, et al. Identification of miRNA master regulators in breast cancer. Cells. 2020;9(7). doi:10.3390/cells9071610

14. Zhou H, Chen L, Qin M, et al. An miRNA signature associated with tumor mutation burden in endometrial cancer. Biosci Rep. 2020;40 (11):BSR20203398.

15. Nagy Á, Lánczky A, Menyhárt O, Győrffy B. Validation of miRNA prognostic power in hepatocellular carcinoma using expression data of independent datasets. Sci Rep. 2018;8(1):9227. doi:10.1038/ s41598-018-27521-y

16. Mao S, Lu Z, Zheng S, et al. Exosomal miR-141 promotes tumor angiogenesis via KLF12 in small cell lung cancer. $J$ Exp Clin Cancer Res. 2020;39(1):193. doi:10.1186/s13046-020-01680-1

17. Travis WD, Brambilla E, Noguchi M, et al. International association for the study of lung cancer/American thoracic society/European respiratory society international multidisciplinary classification of lung adenocarcinoma. $J$ Thorac Oncol. 2011;6(2):244-285. doi:10.1097/JTO.0b013e318206a221

18. Cai M, Shao W, Yu H, Hong Y, Shi L. Paeonol inhibits cell proliferation, migration and invasion and induces apoptosis in hepatocellular carcinoma by regulating miR-21-5p/KLF6 axis. Cancer Manag Res. 2020;6:5931-5943. doi:10.2147/CMAR.S254485

19. Lu K, Han W, Lu K. Identification of key microRNAs involved in tumorigenesis and prognostic microRNAs in breast cancer. Math Biosci Eng. 2020;17(4):2923-2935. doi:10.3934/mbe.2020164

20. Lu M, Kong X, Wang H, Huang G, Ye C, He Z. A novel microRNAs expression signature for hepatocellular carcinoma diagnosis and prognosis. Oncotarget. 2017;8(5):8775-8784. doi:10.18632/ oncotarget.14452

21. Zhang J, Chong CC, Chen GG, Lai PB. A seven-microRNA expression signature predicts survival in hepatocellular carcinoma. PLoS One. 2015;10(6):e0128628. doi:10.1371/journal.pone.0128628 
22. Qin L, Huang J, Wang G, et al. Integrated analysis of clinical significance and functional involvement of microRNAs in hepatocellular carcinoma. $J$ Cell Physiol. 2019;234(12):23581-23595. doi:10.1002/jcp. 28927

23. Chen W, Gao C, Liu Y, Wen Y, Hong X, Huang Z. Bioinformatics analysis of prognostic miRNA signature and potential critical genes in colon cancer. Front Genet. 2020;11:478.

24. Kim JS, Chun SH, Park S, et al. Identification of novel microRNA prognostic markers using cascaded $\mathrm{Wx}$, a neural network-based framework, in lung adenocarcinoma patients. Cancers. 2020;12(7):1890.

25. Denisenko TV, Budkevich IN, Zhivotovsky B. Cell death-based treatment of lung adenocarcinoma. Cell Death Dis. 2018;9(2):117. doi:10.1038/s41419-017-0063-y

26. Peng LN, Deng XY, Gan XX, et al. Targeting of TLE3 by miR-3677 in human breast cancer promotes cell proliferation, migration and invasion. Oncol Lett. 2020;19(2):1409-1417. doi:10.3892/ol.2019.11241

27. Li Y, Zhou Y, Ma L, Liu D, Dai Z, Shen J. miR-3677-3p promotes hepatocellular carcinoma progression via inhibiting GSK3 $\beta$. Acta Biochim Biophys Sin. 2020;52(12):1404-1412. doi:10.1093/abbs/ gmaa125

28. Yao B, Li Y, Niu Y, et al. Hypoxia-induced miR-3677-3p promotes the proliferation, migration and invasion of hepatocellular carcinoma cells by suppressing SIRT5. J Cell Mol Med. 2020;24 (15):8718-8731. doi:10.1111/jcmm. 15503
29. Yu N, Yong S, Kim HK, et al. Identification of tumor suppressor miRNAs by integrative miRNA and mRNA sequencing of matched tumor-normal samples in lung adenocarcinoma. Mol Oncol. 2019;13 (6):1356-1368. doi:10.1002/1878-0261.12478

30. Arias N, Aguirre L, Fernández-Quintela A, et al. MicroRNAs involved in the browning process of adipocytes. $J$ Physiol Biochem. 2016;72(3):463-473. doi:10.1007/s13105-015-0459-Z

31. Yao C, Ruan JW, Zhu YR, et al. The therapeutic value of the SphK1-targeting microRNA-3677 in human osteosarcoma cells. Aging. 2020;12(6):5399-5410. doi:10.18632/aging.102961

32. He Z, Guo X, Tian S, et al. MicroRNA-137 reduces stemness features of pancreatic cancer cells by targeting KLF12. J Exp Clin Cancer Res. 2019;38(1):126. doi:10.1186/s13046-019-1105-3

33. Mak CS, Yung MM, Hui LM, et al. MicroRNA-141 enhances anoikis resistance in metastatic progression of ovarian cancer through targeting KLF12/Sp1/survivin axis. Mol Cancer. 2017;16(1):11. doi:10.1186/s12943-017-0582-2

34. Jia C, Zhang Y, Xie Y, et al. miR-200a-3p plays tumor suppressor roles in gastric cancer cells by targeting KLF12. Artif Cells Nanomed Biotechnol. 2019;47(1):3697-3703. doi:10.1080/21691401.2019. 1594857

\section{Publish your work in this journal}

Cancer Management and Research is an international, peer-reviewed open access journal focusing on cancer research and the optimal use of preventative and integrated treatment interventions to achieve improved outcomes, enhanced survival and quality of life for the cancer patient.
The manuscript management system is completely online and includes a very quick and fair peer-review system, which is all easy to use. Visit http://www.dovepress.com/testimonials.php to read real quotes from published authors. 